\title{
Evaluation of anti-inflammatory and antioxidant for chrysanthemum stem and leaf in zebrafish inflammatory bowel disease model and identification of the bioactive compositions by UPLC-TQ/MS
}

\section{yi Li ( $D$ liyi9823@163.com )}

Nanjing University of Chinese Medicine https://orcid.org/0000-0001-6280-1880

Xiajin Liu

Nanjing University of Traditional Chinese Medicine - Xianlin Campus: Nanjing University of Chinese Medicine

\section{Shulan Su}

Nanjing University of Traditional Chinese Medicine - Xianlin Campus: Nanjing University of Chinese Medicine https://orcid.org/0000-0002-2008-5887

Hui Yan

Nanjing University of Traditional Chinese Medicine - Xianlin Campus: Nanjing University of Chinese Medicine

\section{Sheng Guo}

Nanjing University of Traditional Chinese Medicine - Xianlin Campus: Nanjing University of Chinese Medicine

\section{Dawei Qian}

Nanjing University of Traditional Chinese Medicine - Xianlin Campus: Nanjing University of Chinese Medicine

JinAo Duan

Nanjing University of Traditional Chinese Medicine - Xianlin Campus: Nanjing University of Chinese Medicine

\section{Research Article}

Keywords: Zebrafish, TCM, Inflammatory bowel disease (IBD), UPLC-TQ/MS, Chrysanthemum stem and leaf

Posted Date: January 11th, 2022

DOI: https://doi.org/10.21203/rs.3.rs-1235241/v1 
License: (c) (i) This work is licensed under a Creative Commons Attribution 4.0 International License. Read Full License 


\section{Evaluation of anti-inflammatory and antioxidant for chrysanthemum} stem and leaf in zebrafish inflammatory bowel disease model and identification of the bioactive compositions by UPLC-TQ/MS

Yi Li, Xiajin Liu, Shulan Su *, Hui Yan, Sheng Guo, Dawei Qian, JinAo

$$
\text { Duan * }
$$

Jiangsu Collaborative Innovation Center of Chinese Medicinal Resources Industrialization, National and Local Collaborative Engineering Center of Chinese Medicinal Resources Industrialization and Formulae Innovative Medicine, and Jiangsu Key Laboratory for High Technology Research of TCM Formulae, Nanjing University of Chinese Medicine, Nanjing 210023, China

*Correspondence: Professor Shulan Su and Jinao Duan, College of Nanjing University of Chinese Medicine, Nanjing 210023, China

E-mail addresses: sushulan1974@163.com (S.-L. Su), dja@njucm.edu.cn (J.-A. Duan). 


\begin{abstract}
:
Background: Modern studies have shown that chrysanthemum has anti-inflammatory, regulating intestinal function and other effects, chrysanthemum stem and leaf as a nonmedicinal part of chrysanthemum, has similar chemical components with chrysanthemum, so it is speculated that chrysanthemum stem and leaf also has the effect of regulating intestinal inflammation. The purpose of this study was to evaluate the antiinflammatory and antioxidant effect of chrysanthemum stem and leaf extract through zebrafish inflammatory bowel disease model, and to detect flavonoids, phenolic acids and polysaccharides in chrysanthemum stem and leaf extract.
\end{abstract}

Methods: DSS induced inflammatory bowel disease model of zebrafish was used. Aliciin blue staining was used to observe the secretion of intestinal acid mucin, and H\&E staining was used to detect the inflammatory cell infiltration. Superoxide dismutase activity was determined by kit, and the expression levels of key inflammatory cytokines IL-1 $\beta$, IL8 and MMP9 were detected by quantitative polymerase chain reaction. Furthermore, UPLC-TQ /MS method was used to detect the contents of flavonoids and phenolic acids in chrysanthemum stem and leaf extracts. Neutral and acidic polysaccharides were determined by the phenol-sulfuric acid method and the carbazol-sulfuric acid method.

Results: H\&E staining showed that extracts from chrysanthemum stem and leaf inhibited inflammatory cell infiltration to varying degrees. The expressions of inflammatory cytokines IL-1 $\beta$, IL8 and MMP9 were significantly increased in DSS induced zebrafish. The extracts inhibited the expression of IL-1 $\beta$, IL8 and MMP9 in DSS induced zebrafish. The water extract $0.2 \mathrm{mg} / \mathrm{mL}$ and alcohol extract $0.04 \mathrm{mg} / \mathrm{mL}$ had the most significant inhibition. Superoxide dismutase activity in extract treatment group was also up-regulated compared with model group. The results showed that the contents of total flavonoids and phenolic acids in the alcohol extract of chrysanthemum stem and leaf were significantly higher than those in the water extract of chrysanthemum stem and leaf, but the water-soluble polysaccharides were significantly more in the water extract of chrysanthemum stem and leaf.

Conclusions: In conclusion, this study suggests that chrysanthemum stem and leaf 
extract can improve inflammatory bowel disease of zebrafish through antiinflammatory and antioxidant activities.

Keywords: Zebrafish, TCM; Inflammatory bowel disease (IBD), UPLC-TQ/MS, Chrysanthemum stem and leaf 


\section{Introduction}

Inflammatory bowel disease (IBD) includes Crohn's disease (CD) and ulcerative colitis (UC). Symptoms can include abdominal pain, vomiting, diarrhea, and weight loss ${ }^{1-3}$. With the incidence and prevalence of IBD increasing over time in different parts of the world, the disease has now become a global disease ${ }^{4-6}$. Some studies show that about 6.8 million people worldwide suffer from $\mathrm{IBD}^{7}$. In recent years, the pathogenesis of IBD has made some progress, involving environmental factors, psychological factors, genetic factors and intestinal microbes ${ }^{8,9}$, but the specific pathogenesis is not clear. The main manifestations of the patient are abdominal pain, diarrhea, fever, weight loss, and possible sub-occlusion (spasm pain, vomiting, difficulty with faeces / discharge) or other complications (i.e., abscess, fistula) ${ }^{10}$. At present, western medicine treatment drugs and means have been more common, such as corticosteroids, salicylate and antibiotics $^{11}$, these drugs can significantly reduce inflammation, improve clinical symptoms, but the long-term use of still has certain side effects ${ }^{12,13}$, greatly reduce the patient's life quality and satisfaction. Therefore, looking for a safe and effective treatment is particularly important. Traditional Chinese medicine (TCM) has long been used to treat many clinical diseases, such as diarrhea, infection and functional disorders 14. TCM emphasizes the treatment based on syndrome differentiation, and TCM has less side effects and significant efficacy, so it is increasingly used in clinical practice ${ }^{15}$.

Flos Chrysanthemi (Chrysanthemum) is the dry flower head of Chrysanthemum morifolium Ramat. As a kind of Traditional Chinese medicine (TCM), it has been widely used in wind-heat cold, headache and dizziness, eye swelling and pain, eye coma, sore carbuncle and toxin. The pharmacological effects of modern studies include antibacterial, anti-inflammatory ${ }^{16}$, antioxidant and regulation of intestinal dysfunction, is a good alternative to antibiotics. Studies have also shown that chrysanthemum polysaccharide can improve colitis and functional constipation in rats by regulating intestinal microbes ${ }^{17-20}$. Its main bioactive components include flavonoids, polysaccharides, phenolic acids, volatile oils and terpenoids ${ }^{21-23}$, and the main chemical components in chrysanthemum stem and leaf are very similar to chrysanthemum, so it 
is speculated that chrysanthemum stem and leaf have similar functions in regulating gastrointestinal function ${ }^{24,25}$.

Zebrafish (Danio rerio) is a tropical ornamental fish belonging to the Genus Danil of cyprinidae. The adult fish of zebrafish is small, easy to raise, rapid development, short sexual maturity, strong reproduction, and transparent embryo development in vitro, which makes it easy to observe. These characteristics make zebrafish widely used in high-throughput screening. Since the 1980s, zebrafish has received attention from the field of genetics and biology, and has gradually become one of the important model vertebrates ${ }^{26}$. With the completion of zebrafish gene sequencing, zebrafish genome sequence and human gene conserved as high as $70 \%{ }^{27}$, which means that zebrafish experimental results can also be applied to humans, so zebrafish is increasingly widely used in the study of human diseases.

Therefore, in this study, intestinal dysfunction model was established by inducing intestinal injury of zebrafish with sodium glucan sulfate (DSS), and the intervention effect of chrysanthemum stem and leaf on inflammatory bowel disease was evaluated. The contents of total phenolic acids and total flavonoids in chrysanthemum stem and leaf were determined by ULTRA-performance liquid chromatography-triple quadrupole electrospray tandem mass spectrometry (UPLC-TQ/MS). Neutral and acidic polysaccharides were determined by phenol-sulfuric acid method ${ }^{28}$ and carbazolsulfuric acid method ${ }^{29}$.

\section{Materials and methods}

\section{Animal care ethics}

All zebrafish experiments were conducted according to the guidelines of Animal Ethics Committee of the Laboratory Animal Center, Nanjing University of Traditional Chinese Medicine.

\section{Zebrafish husbandry}

The adult AB strain zebrafish (YSY Biotechnology Inc., Nanjing, China), raised in the dark/light $(10 \mathrm{~h}: 14 \mathrm{~h})^{30}, 26^{\circ} \mathrm{C}$ environment, live brine shrimp feed is cast twice a 
day. One day before the experiment, healthy zebrafish with reproductive ability were placed in spawning boxes with a 1:1 or 1:2 male to female ratio, separated by a partition. The partitions were pulled out at 9:00 the next morning, and the fertilized eggs were collected in the box at 11:30. The collected embryos were placed in a constant temperature incubator at $28^{\circ} \mathrm{C}$ and kept under $14 \mathrm{~h}$ light. E3 culture water ${ }^{31}(5 \mathrm{mM} \mathrm{NaCl}$, $0.17 \mathrm{~mm} \mathrm{KCl}_{1} 0.33 \mathrm{~mm} \mathrm{CaCl}_{2}, 0.33 \mathrm{~mm} \mathrm{MgSO}_{4}, \mathrm{pH}=7.2$ ) was prepared, and $\mathrm{E} 3$ was used as a specific medium for embryo culture, rather than zebrafish fish culture system water, to ensure stable buffer salt concentration and $p \mathrm{H}$. Dead eggs were removed every 24 hours and fresh embryo culture medium was replaced.

\section{Chemicals and regents}

The stems and leaves of chrysanthemum were collected from Yangma Town, Sheyang County, Yancheng City, Jiangsu Province in November 2019, and extracted and concentrated by Kanion Pharmaceutical using industrial percolation extraction, vacuum concentration and other processes to obtain water extraction (ST) and alcohol extraction (CT) extract respectively.

The control substance isochlorogenic acid $\mathrm{A}$, isochlorogenic acid $\mathrm{B}$, isochlorogenic acid C, cynarin (1,3-dicaffeoylquinic acid), Eriodictyol, Apigenin-7-O- $\beta$-D-glucoside, Cryptochlorogenic acid, Tilianin, Neochlorogenic acid, Diosmetin, Diosmetin-7glucoside were purchased from Shanghai Yuanye Bio-Technology Co., LTD(Shanghai, China). Caffeic acid, Chlorogenic acid, Luteolin and Luteolin 7-O- $\beta$-D-glucoside, Apigenin, Acacetin, Linarin, Hesperetin and so on were purchased from the Nanjing chunqiu Biological Engineering Co., LTD (Nanjing, China). The purity of the above 28 reference substances were more than $98 \%$ by HPLC. HPLC-grade acetonitrile and methanol were from Merk (Darmstadt, Germany). Other chemical reagents purchased from Shanghai Sinopharm Chemical Reagent Co., LTD (Shanghai,China), are analytical pure. Dextran sulfate sodium salt (DSS), molecular weight 36,000-50,000 (Lot Number: S3045, MP, Biomedicals, LLC). Glucuronic acid and Glucose were purchased from Aladdin Biochemical Technology Co., LTD (Shanghai,China).

Deionized water was distilled and purified by an EPED super-purification system 
(Eped, Nanjing, China).

\section{Apparatus}

ACQUITYTM UPLC system, XevoTM TQ mass spectrometry system (Waters Corporation, Milford, USA); Startorius BT1250 electronic balance (Sartorius Scientific Instruments Corporation, Beijing, China); TDL240B centrifuge (Anting Scientific Instrument Corporation, Shanghai, China), WH-1 micro-vortex mixing instrument (Shanghai, China); CENTRIVAP centrifuge enrichment apparatus (Labconco). EPED super-purification system (Eped, Nanjing, China).

\section{Sample preparation}

The standard stock solution of 19 phenolic acids and flavonoids was prepared with methanol as solvent. They were $1.62 \mathrm{mg} / \mathrm{mL}$ for Neochlorogenic acid, $1.90 \mathrm{mg} / \mathrm{mL}$ for Chlorogenic acid, $1.86 \mathrm{mg} / \mathrm{mL}$ for Cryptochlorogenic acid, $1.46 \mathrm{mg} / \mathrm{mL}$ for $1,3-$ dicaffeoylquinic acid, $1.96 \mathrm{mg} / \mathrm{mL}$ for Isochlorogenic acid $\mathrm{B}, 1.72 \mathrm{mg} / \mathrm{mL}$ for Isochlorogenic acid $\mathrm{A}, 1.85 \mathrm{mg} / \mathrm{mL}$ for Isochlorogenic acid $\mathrm{C}, 1.74 \mathrm{mg} / \mathrm{mL}$ for Caffeic acid, $1.10 \mathrm{mg} / \mathrm{mL}$ for Luteolin-7- $O-\beta$-D-glucoside, $0.90 \mathrm{mg} / \mathrm{mL}$ for Apigenin-7-Oglucoside, $1.07 \mathrm{mg} / \mathrm{ml}$ for Diosmetin-7-glucoside, $1.10 \mathrm{mg} / \mathrm{mL}$ for Eriodictyol, $1.03 \mathrm{mg} / \mathrm{mL}$ for Linarin, $0.78 \mathrm{mg} / \mathrm{mL}$ for Tilianin, $1.29 \mathrm{mg} / \mathrm{mL}$ for Apigenin, $1.12 \mathrm{mg} / \mathrm{mL}$ for Diosmetin, $1.05 \mathrm{mg} / \mathrm{mL}$ for Hesperetin, $1.00 \mathrm{mg} / \mathrm{mL}$ for Acacetin and $1.26 \mathrm{mg} / \mathrm{mL}$ for Luteolin.

Then appropriate amount of reserve solution of 8 phenolic acids and 11 flavonoids reference substances was taken respectively, and methanol was added to make two mixed reference substance solutions containing each reference substance in the appropriate concentration range. The final concentration of phenolic acids were: $135 \mu$ $\mathrm{g} / \mathrm{mL}$ for Neochlorogenic acid, $158 \mu \mathrm{g} / \mathrm{mL}$ for Chlorogenic acid, $157 \mu \mathrm{g} / \mathrm{mL}$ for Cryptochlorogenic acid, $122 \mu \mathrm{g} / \mathrm{mL}$ for 1,3 -dicaffeoylquinic acid, $163 \mu \mathrm{g} / \mathrm{mL}$ for Isochlorogenic acid $\mathrm{B}, 143 \mu \mathrm{g} / \mathrm{mL}$ for Isochlorogenic acid $\mathrm{A}, 154 \mu \mathrm{g} / \mathrm{mL}$ for Isochlorogenic acid C, $145 \mu \mathrm{g} / \mathrm{mL}$ for Caffeic acid. The final concentration of flavonoids were: $69 \mu \mathrm{g} / \mathrm{mL}$ for Luteolin-7-O- $\beta$-D-glucoside, $56 \mu \mathrm{g} / \mathrm{mL}$ for Apigenin-7-O-glucoside, $89 \mu \mathrm{g} / \mathrm{mL}$ for Diosmetin-7-glucoside, $69 \mu \mathrm{g} / \mathrm{mL}$ for Eriodictyol, $64 \mu \mathrm{g} / \mathrm{mL}$ for Linarin, 
$49 \mu \mathrm{g} / \mathrm{mL}$ for Tilianin, $81 \mu \mathrm{g} / \mathrm{mL}$ for Apigenin, $70 \mu \mathrm{g} / \mathrm{mL}$ for Diosmetin, $66 \mu \mathrm{g} / \mathrm{mL}$ for Hesperetin, $62.5 \mu \mathrm{g} / \mathrm{mL}$ for Acacetin and $79 \mu \mathrm{g} / \mathrm{mL}$ for Luteolin. A series of mass concentrations were prepared by successive dilution, which were used to examine the linear relationship. The mixed reference solution with different concentrations was filtered by $0.22 \mu \mathrm{m}$ microporous membrane and stored at $4^{\circ} \mathrm{C}$ for later use. Appropriate amount of Glucose and Glucuronic acid reference substance was taken, weighed accurately and placed in a $10 \mathrm{~mL}$ volumetric flask. After dissolved in pure water, diluted to scale and mixed, the glucose and glucuronic acid reference stock solutions with concentrations of $0.195 \mathrm{mg} / \mathrm{mL}$ and $0.195 \mathrm{mg} / \mathrm{mL}$ were obtained, and stored at $4{ }^{\circ} \mathrm{C}$ for later use.

Water extract and alcohol extract extracted from industry were taken, and the yield was measured after freeze-drying. The freeze-dried yield of water extract and alcohol extract was $16.13 \%$ and $16.94 \%$ respectively. Weigh the water-extracted freeze-dried powder and alcohol-extracted freeze-dried powder about 1mg each, weigh them accurately and place them in a $1 \mathrm{ml}$ volumetric bottle, add methanol to a constant volume to scale, and mix well. Centrifuged at $13000 \mathrm{r} \cdot \mathrm{min}^{-1}$ for $15 \mathrm{~min}$ before injection, the supernatant was filtered through $0.22 \mu \mathrm{m}$ microporous membrane, and the filtrate was used as the test solution, and stored at $4^{\circ} \mathrm{C}$ for later use.

\section{Zebrafish IBD modeling and drug treatment}

Dextran Sulfate Sodium (DSS) is a sulfated polysaccharide that can cause chemical damage to intestinal mucosa. Zebrafish model with intestinal dysfunction was established using $0.5 \% \mathrm{DSS}^{32}$ and the concentration was screened. The water extract and alcohol extract of chrysanthemum stem and leaf were prepared with different concentrations. According to the screening results, the concentration of alcohol extract from chrysanthemum stem and leaf was determined to be $0.02,0.04,0.06 \mathrm{mg} / \mathrm{mL}$. The concentration of water was $0.2,0.4$ and $0.6 \mathrm{mg} / \mathrm{mL}$. $40-50$ juvenile 3 days postfertilization(dpf) fish in each group were divided into blank group, model group (immersed in $0.5 \%$ DSS), alcohol-extract group (immersed in DSS+ immersed in 
alcohol-extract of chrysanthemum stem and leaf), and water-extract group (immersed in DSS+ immersed in water extract of chrysanthemum stem and leaf). Each group was administered for $72 \mathrm{~h}$ for three times in parallel, and the solution was changed every $24 \mathrm{~h}$ to observe the survival rate.

\section{Histological analysis}

\section{Alcian blue staining}

DSS induced intestinal injury in zebrafish leads to accumulation of acidic mucin in intestinal bulb. Moreover, a significant feature of DSS modeled zebrafish is alcian blue staining in intestinal globules, and the accumulation of acidic mucin in intestinal globules is called "mucinous phenotype". In order to observe the changes of intestinal goblet cells in THE DSS model, alcian blue staining of acidic mucin was performed for comparison between the model group and the blank group ${ }^{32}$. Specific dyeing methods are as follows:

(1) The young fish were fixed in $4 \%$ paraformaldehyde at room temperature for 2 hours.

(2) Rinse the young fish with acidic ethanol (70\% ethanol, $1 \%$ concentrated hydrochloric acid).

(3) Soak young fish in Alcian blue staining solution (0.001\% Alcian blue, 80\% ethanol, $20 \%$ glacial acetic acid) for $15 \mathrm{~min}$ at room temperature.

(4) Remove the staining solution and rinse the background repeatedly with acidic ethanol.

(5) The young fish were placed in 3\% methylcellulose for imaging. Staining samples in acidic ethanol should be stored in sealed containers to avoid dehydration due to evaporation.

\section{Hematoxylin eosin staining}

$6 \mathrm{dpf}$ zebrafish embryos were immobilized overnight in 4\% PFA at room temperature and then progressively dehydrated in ethanol. The embryos were embedded in paraffin sections, cut into $3 \mu \mathrm{m}$ sections, and stained with hematoxylin-eosin. At least three embryos were examined under each treatment condition. 


\section{Superoxide dismutase (SOD)}

SOD is a key enzyme in the antioxidant system, which can inhibit oxidative damage caused by external stimuli to the body, and the level of SOD can reflect the degree of the body's anti-inflammatory response. $72 \mathrm{~h}$ later, 25 juvenile zebrafish in each group were collected and washed in $1.5 \mathrm{ml}$ centrifuge tube with normal saline for 3 times, and treated with 1:19 (zebrafish quality: (normal saline volume), 5\% tissue homogenate was prepared by automatic sample rapid grinding machine, centrifuged at $4{ }^{\circ} \mathrm{C}$ for 5000 $\mathrm{RPM} \times 15 \mathrm{~min}$, and supernatant was taken.

Before the test, $5 \%$ tissue homogenate was diluted into different concentrations for pre-experiment, and the concentration with inhibition rate of $40-60 \%$ was selected. The pre-experiment results showed that the inhibition rate of $1 \%$ tissue homogenate met the requirements. The protein concentration of $1 \%$ tissue homogenate was determined according to the BCA method protein concentration determination kit. The activity of superoxide dismutase (SOD) in $1 \%$ tissue homogenate of each group was detected according to the instruction of SOD assay kit.

Calculation formula:

(1) SOD inhibition rate $(\%)=($ A control $-\mathrm{A}$ control blank $)-($ A determination $-\mathrm{A}$ determination blank $) /(($ A control -A control blank $)) \times 100 \%$

(2) SOD activity $(\mathrm{U} / \mathrm{mgprot})=\mathrm{SOD}$ inhibition rate $\div 50 \% \times$ dilution ratio of reaction system $\div$ protein concentration of samples to be tested (mgprot $/ \mathrm{mL})$

\section{RNA extraction and q-PCR}

The 6 dpf zebrafish tissues were collected and put into a homogenizer, and RNA of each sample was extracted. The RNA of each group was reverse transcribed to obtain cDNA. The expression levels of genes related to inflammation were determined by bioRAD CFX96 real-time system. Results $\beta$-actin was used as internal reference. PCR primers of interleukin (IL) -1 $\beta$, IL8, Matrix metallopeptidase 9(MMP9) and reference gene $\beta$-actin were synthesized and purified by Shanghai Sangon Bio-Technology Co., LTD (Shanghai, China), and their quality was detected. Primer information of RTQPCR is shown in Table 5. 
After the RT-QPCR reaction, the original data were derived. Import the original data into Excel, and use $2^{-\Delta \Delta \mathrm{Ct}}$ calculation method for data processing. SPSS software was used to conduct one-way ANOVA, and $P<0.05$ indicated significant difference. GraphPad 8 software was used to draw experimental data pictures.

Table 5 Primer information of RT-QPCR

\begin{tabular}{ccc}
\hline Gene & Forward primer sequence & Reverse primer sequence \\
\hline Il8 & GTCGCTGCATTGAAACAGAA & CTTAACCCATGGAGCAGAGG \\
mmp9 & CTTCAAGGACGGGCGCTACT & GAGGTGGTCCTCAAAGGCAG \\
Il1 $\beta$ & TGGACTTCGCAGCACAAAATG & GTTCACTTCACGCTCTTGGATG \\
$\beta$-actin & CACACCGTGCCCATCTATGA & TTCTCTTTCGGCTGTGGTGG \\
\hline
\end{tabular}

UPLC-TQ/MS analysis

\section{UPLC-TQ/MS conditions}

A Waters Acquity UPLC BEH C 18 column $\left(2.1 \times 100 \mathrm{~mm}^{2}, 1.7 \mu \mathrm{m}\right.$, Waters Corporation, Milford, USA) was used with a flow rate of $0.4 \mathrm{~mL} \cdot \mathrm{min}^{-1}$. The column temperature was $30^{\circ} \mathrm{C}$, the automatic sampler temperature was $10^{\circ} \mathrm{C}$, and the sample volume was $2 \mu \mathrm{L}$. The mobile phase gradient program was $0.1 \%$ formic acid water (A) and acetonitrile (B): 0-3 min, 4-7\% B; 3-6 min, 7\% B; 6-7 min, 7-15\% B; 7-14 min, 15\% B; 14-17 min, $15-35 \% \mathrm{~B} ; 17-21 \mathrm{~min}, 35-100 \% \mathrm{~B}$.

Electrospray ion source (ESI) was used. Simultaneous detection of positive and negative ions; IonSpray Voltage in positive and negative ion modes: $5500 \mathrm{~V} /-4500 \mathrm{~V}$; Ion source atomization temperature: $400^{\circ} \mathrm{C}$; Curtain Gas: 30 psi; Spray gas (Ion Source Gas1): 40 psi; Auxiliary heating gas (Ion Source Gas2): 40 psi; Scanning method: multiple reactive ion monitoring (MRM).

Linearity

The preparation solution of mixed reference substance was diluted to prepare mixed reference substance solution with a series of mass concentrations. UPLC-TQ /MS analysis was performed according to the chromatographic conditions and the mass 
spectrometric conditions. With the peak area as $\mathrm{Y}$ and the concentration of reference solution $\left(\mathrm{ng} \cdot \mathrm{mL}^{-1}\right)$ as $\mathrm{X}$, linear regression analysis was performed to calculate the correlation coefficient.

\section{Precision and accuracy}

Precision test: the mixed reference solution was injected 6 times in one day to determine the peak area of each component to be measured, and the intraday precision was evaluated by the relative standard deviation (RSD \%) of each peak area.

Repeatability test: take the last 6 samples of freeze-dried powder, each about $1.0 \mathrm{mg}$, weigh accurately, prepare the test solution, and calculate the content and RSD value of each component to be measured in the test solution after UPLC-TQ /MS analysis.

Stability test: a sample solution from the repeatability test was injected into the liquid chromatograph at $0,2,4,8,12$ and 24h, respectively. After the UPLC-TQ /MS analysis, the peak area and RSD values of each component to be measured were calculated.

\section{Data analysis of UPLC-TQ/MS}

MassLynx4.2 (Waters Corporation, Milford, USA) software was used to analyze the contents of total flavonoids and phenolic acids in water extract and alcohol extract of chrysanthemum stem and leaf, and SPSS22.0 was used for statistical analysis. Independent sample $\mathrm{T}$ test was used for comparison. A value of $\mathrm{P}<0.05$ was considered significant.

\section{Determination of total polysaccharide content}

Using Glucose and Glucuronic acid as control, the neutral polysaccharide content in chrysanthemum stem and leaf was determined by phenol-sulfuric acid method, and the acid polysaccharide content in chrysanthemum stem and leaf was determined by carbazol-sulfuric acid method. Total polysaccharide content $=$ neutral polysaccharide content + acid polysaccharide content.

\section{Results}

\section{Pathological changes in DSS-induced enterocolitis}

\section{Alcian blue staining}


Staining results showed that the secretion of acidic mucin in the DSS treated group was significantly increased compared with that in the untreated group, indicating that the DSS induced inflammatory bowel disease model was successful (Figure 1).

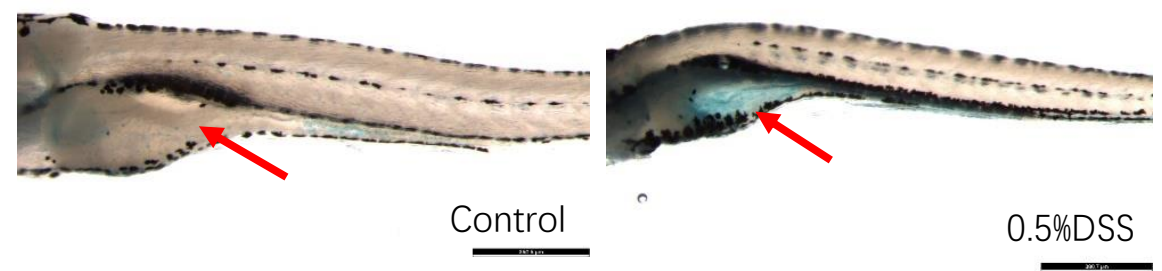

A

B

Figure 1. Alcian blue staining of zebrafish in blank group and $0.5 \% \mathrm{DSS}$ model group

(A) control group, (B) $0.5 \% \mathrm{DSS}$ model group, whole alcian blue staining, red arrow shows intestinal acid mucin secretion.

\section{Hematoxylin eosin staining}

After pathological sections were stained and observed under microscope, no obvious lesions were observed in the blank group, and enlarged gut lumen and disrupted epithelial layer in zebrafish larvae treated with DSS, suggesting decreased peristalsis and impaired mucosal barrier were observed in the intestinal lumen $72 \mathrm{~h}$ after modeling with $0.5 \%$ DSS. Compared with the model group, the intestinal mucosa degeneration in the water and alcohol extraction groups was lower. The staining results are shown in Figure 2. 

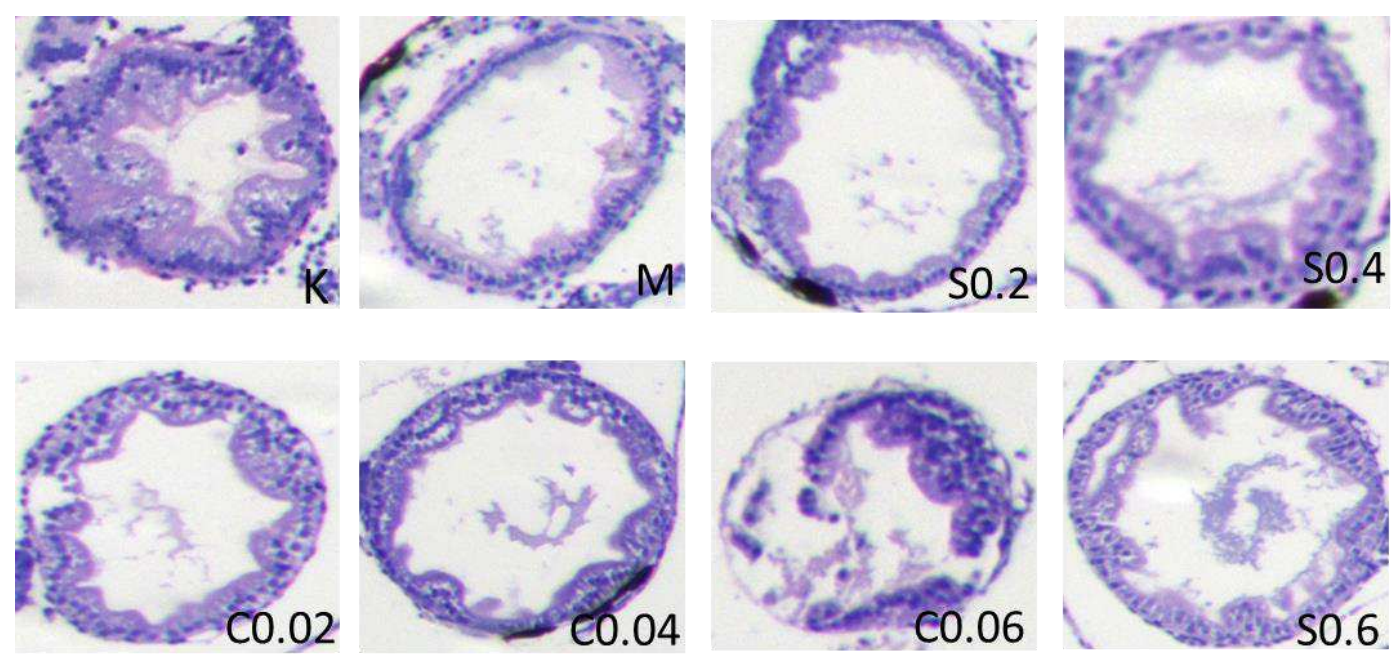

Figure 2. HE staining results of intestinal cross-section of zebrafish in blank group, model group and administration group

\section{Changes in superoxide dismutase levels during DSS induced inflammation}

The model was made by $0.5 \%$ DSS, and the concentration of $0.02,0.04,0.06 \mathrm{mg} / \mathrm{mL}$ alcohol extract of chrysanthemum stem and leaf and $0.1,0.2,0.4 \mathrm{mg} / \mathrm{mL}$ water extract of chrysanthemum stem and leaf were given to determine the corresponding SOD value. SOD activity in model group was significantly lower than that in blank group $(P<0.01)$. Compared with the model group, SOD activity in the alcohol extract groups of 0.02 , 0.04 and $0.06 \mathrm{mg} / \mathrm{mL}$ and the water extract groups of $0.1,0.2$ and $0.4 \mathrm{mg} / \mathrm{mL}$ of chrysanthemum stem and leaf increased with the increase of drug concentration. There was no significant difference between the $0.1 \mathrm{mg} / \mathrm{mL}$ water extract administration group and the model group, while there were significant or extremely significant differences between the other groups and the model group, and the increase of SOD in the $0.06 \mathrm{mg} / \mathrm{mL}$ alcohol extract group and $0.4 \mathrm{mg} / \mathrm{mL}$ water extract group was close to that in the blank group, as shown in Figure 3.

It was concluded that $0.02,0.04$ and $0.06 \mathrm{mg} / \mathrm{mL}$ alcohol extract groups and 0.2 and $0.4 \mathrm{mg} / \mathrm{ml}$ water extract groups of chrysanthemum stem and leaf effectively inhibited intestinal injury of zebrafish, and played a certain therapeutic effect on intestinal dysfunction induced by DSS. In conclusion, both alcohol and water extracts from chrysanthemum stem and leaf can inhibit oxidative damage of intestinal dysfunction zebrafish by changing the activity of superoxide dismutase (SOD), and play a certain 
role in regulating intestinal dysfunction.

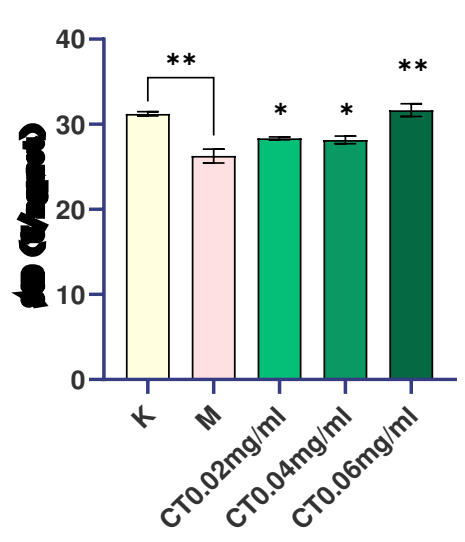

A

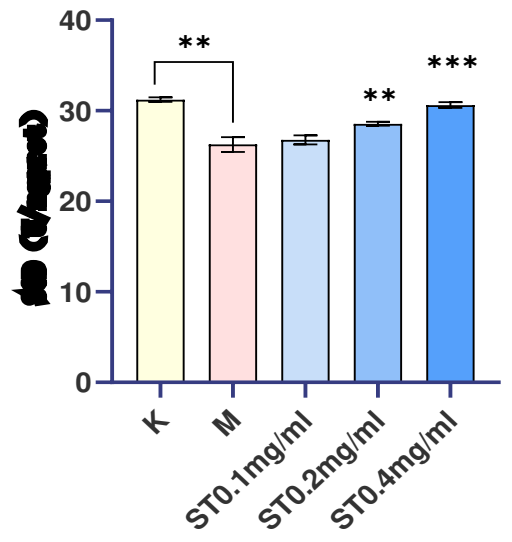

B

Figure. 3 Comparison of SOD activity between blank group, model group and chrysanthemum stem and leaf administration group

A: Changes of SOD activity after modeling and alcohol extract of chrysanthemum stem and leaf were administered; B: Changes of SOD activity after modeling and administration of alcohol extract from chrysanthemum stem and leaf. $(P<0.05$,

$$
* * P<0.01, * * * P<0.001) .
$$

\section{Inflammatory cytokine production in larvae exposed to DSS}

The proinflammatory cytokines IL-1 $\beta$, IL8 and MMP9 were selected in this study, we then measured the expression levels of IL-1 $\beta$, IL8 and MMP9 using q-PCR. As shown in Figure 4, Compared with blank group, mRNA expressions of IL-1 $\beta$, IL8 and MMP9 genes in model group were significantly increased $(P<0.001)$, indicating that there was inflammation in model group. Compared with model group, the up-regulation of IL-1 $\beta$, IL8 and MMP9 induced by DSS was significantly inhibited in water extract group and alcohol extract group $(P<0.05,0.01,0.001)$, and the inhibition effect of S0.2 in water extract group and $\mathrm{C} 0.04$ in alcohol extract group was the most significant $(P<$ 0.001). In conclusion, these results suggest that water and alcohol extracts from chrysanthemum stem and leaf have a protective effect on DSS induced inflammatory bowel disease model of zebrafish and inhibit DSS induced inflammatory response. 


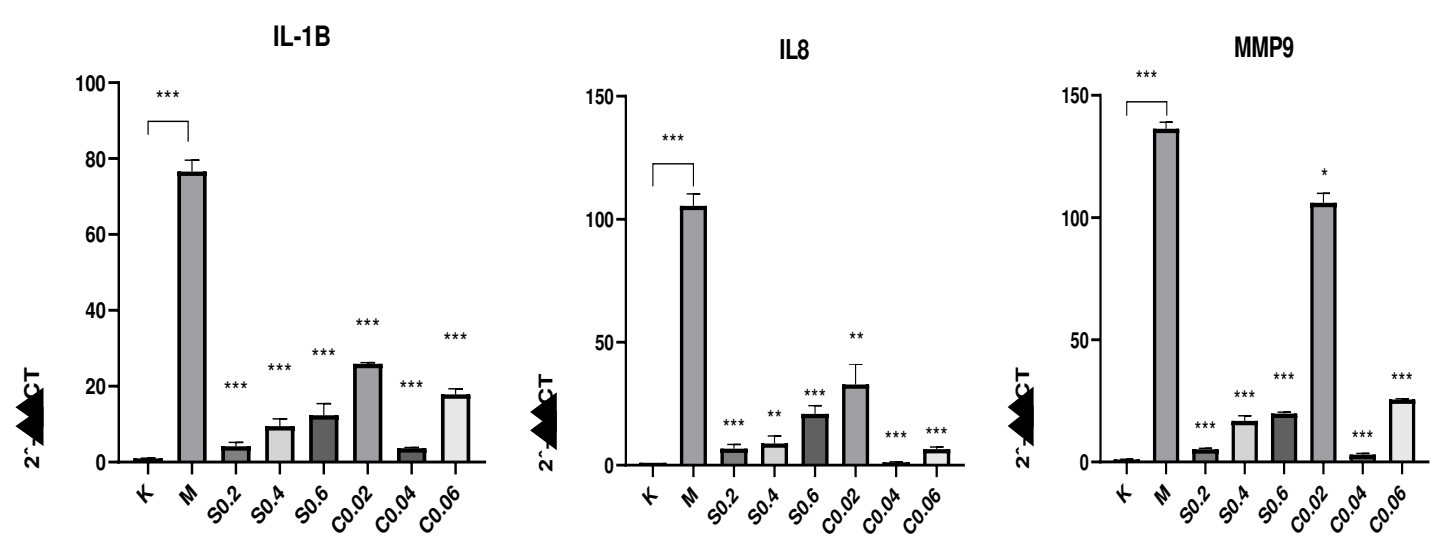

Figure 4. mRNA expression results of related inflammatory factors in blank group, model group and administration group

Compared with model group, $*$ represents $P<0.05, * * \mathrm{P}<0.01, * * * \mathrm{P}<0.001$.

Flavonoids and phenolic acids were determined by UPLC-TQ/MS method

\section{Linearity}

The results showed that the 19 flavonoids and phenolic acids showed a good linear relationship in the determination range. The regression equation, $\mathrm{R}^{2}$ and linear range of 19 reference substances were given in Table 6. The TQ chromatogram of each component was shown in Figure 5.

Table 6 Linear relationship between peak area and concentration of reference substance

\begin{tabular}{ccccc}
\hline Number & Analytes & Calibration curves ${ }^{a}$ & $\mathrm{R}^{2}$ & $\begin{array}{c}\text { Linear range } \\
(\mathrm{ng} / \mathrm{ml})\end{array}$ \\
\hline 1 & Neochlorogenic acid & $\mathrm{y}=20367 \mathrm{x}+14658$ & 0.9987 & $13.5-135000.0$ \\
2 & Chlorogenic acid & $\mathrm{y}=14377 \mathrm{x}+12830$ & 0.9975 & $15.8-158000.0$ \\
3 & Caffeic acid & $\mathrm{y}=11510 \mathrm{x}-359.18$ & 0.9928 & $14.5-145000.0$ \\
4 & Cryptochlorogenic & $\mathrm{y}=12772 \mathrm{x}+15468$ & 0.9993 & $15.7-157000.0$ \\
& acid & & & \\
5 & 1,3-dicaffeoylquinic & $\mathrm{y}=44.539 \mathrm{x}+37.236$ & 0.9967 & $12.2-122000.0$ \\
6 & acid & & & \\
\hline
\end{tabular}


glucoside

\begin{tabular}{|c|c|c|c|c|}
\hline 7 & Isochlorogenic acid B & $y=1162.6 x+130.06$ & 0.9999 & $16.3-163000.0$ \\
\hline 8 & Isochlorogenic acid A & $y=41.259 x-9.1657$ & 0.9998 & $14.3-143000.0$ \\
\hline 9 & $\begin{array}{l}\text { Apigenin-7- } O-\beta \text {-D- } \\
\text { glucoside }\end{array}$ & $y=28985 x+2386.8$ & 1 & $5.6-56000.0$ \\
\hline 10 & Isochlorogenic acid C & $y=23.482 x+28.95$ & 0.9978 & $15.4-154000.0$ \\
\hline 11 & $\begin{array}{l}\text { Diosmetin-7- } \\
\text { glucoside }\end{array}$ & $y=10955 x+5588.4$ & 0.9994 & $8.9-89000.0$ \\
\hline 12 & Eriodictyol & $y=35603 x-1415.7$ & 0.9991 & $6.9-69000.0$ \\
\hline 13 & Linarin & $y=7679.9 x+2816$ & 0.9986 & $6.4-64000.0$ \\
\hline 14 & Tilianin & $y=3.7865 x+3.4176$ & 0.9979 & $4.9-49000.0$ \\
\hline 15 & Apigenin & $y=348.4 x-6.4809$ & 0.9997 & $8.1-81000.0$ \\
\hline 16 & Diosmetin & $y=561.49 x-11.501$ & 0.9961 & $7.0-70000.0$ \\
\hline 17 & Hesperetin & $y=90914 x-3254.3$ & 0.9986 & $6.6-66000.0$ \\
\hline 18 & Acacetin & $y=28421 x+8227.3$ & 0.9995 & $6.25-62500.0$ \\
\hline 19 & Luteolin & $y=290.93 x-4.1891$ & 0.998 & 7.9-79000.0 \\
\hline
\end{tabular}

${ }^{a} \mathrm{y}$ is the value of peak area, and $\mathrm{x}$ is the value of the reference compound's concentration $(\mathrm{ng} / \mathrm{mL})$

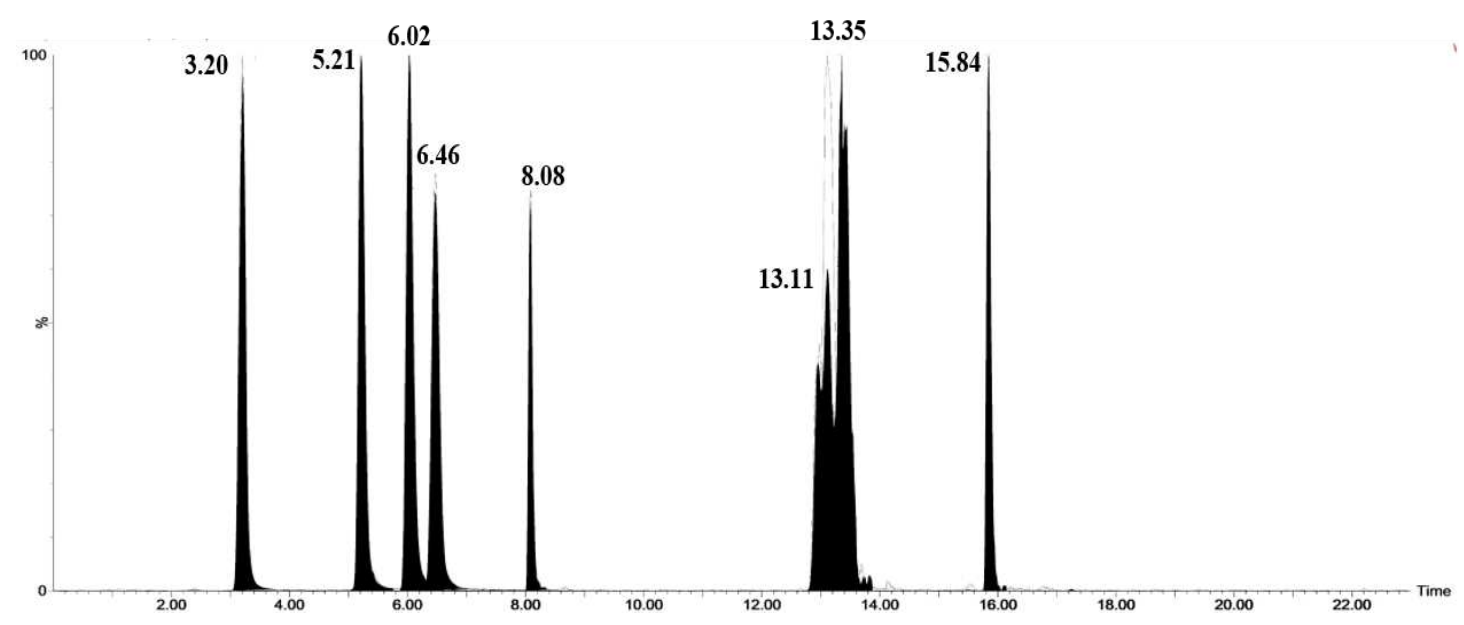




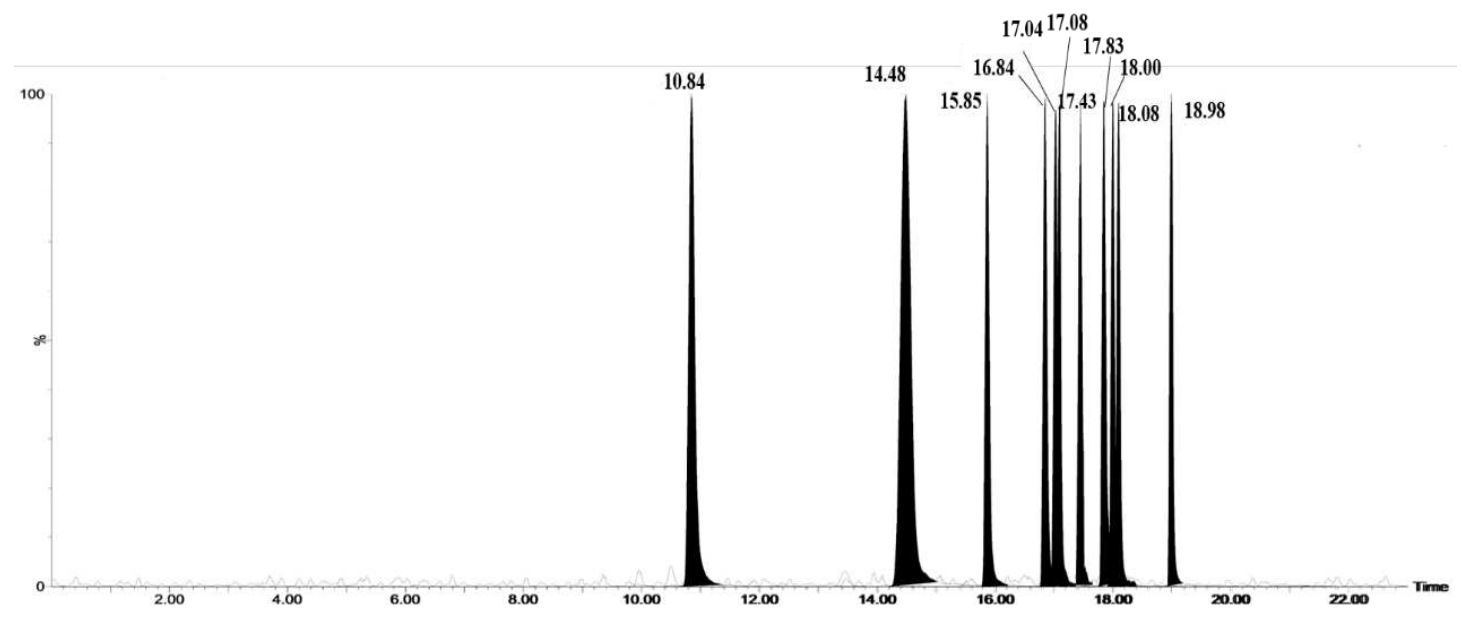

Figure 5 TQ chromatograms of 19 flavonoids and phenolic acids

Notes : According to the peak order, the components are respectively : (A)

Neochlorogenic acid; (B) Chlorogenic acid; (C) Caffeic acid; (D) Cryptochlorogenic acid; (E) 1,3-Dicaffeoylquinic acid; (F) Isochlorogenic acid B; (G) Isochlorogenic acid A; (H) Isochlorogenic acid C; (I) Luteolin-7-O- $\beta$-D-glucoside; (J) Apigenin-7-O$\beta$-D-glucoside; (K) Diosmetin-7-glucoside; (L) Eriodictyol; (M) Luteolin; (N)

Linarin; (O) Tilianin; (P) Apigenin; (Q) Diosmetin; (R) Hesperidin; (S) Actinin. Precision, repeatability and stability

The precision test results showed that the RSD values of 19 phenolic acids and flavonoids were all less than $4.27 \%$, indicating good precision of the instrument. Repeatability test results showed that the repeatability of 19 components in water extract (ST) and alcohol extract (CT) of chrysanthemum stem and leaf was less than $4.75 \%$ and $4.13 \%$, respectively, indicating that the method had good repeatability. Stability test results showed that the stability of 19 components in water extract (ST) and alcohol extract (CT) of chrysanthemum stem and leaf was less than $4.50 \%$ and $4.95 \%$, respectively, indicating that the tested solution had good stability within $24 \mathrm{~h}$.

Table 7 Precision, repeatability and stability test results of 19 phenolic acids and flavonoids

\begin{tabular}{lcccc}
\hline & & Precision & Repeatability & Stability (RSD\%, \\
Number & Analytes & (RSD\% & $(\mathrm{RSD} \%, \mathrm{~N}=6)$ & $\mathrm{N}=6)$ \\
\end{tabular}




\begin{tabular}{|c|c|c|c|c|c|c|}
\hline & & \multirow[b]{2}{*}{$, \mathrm{N}=6)$} & \\
\hline & & & $\mathrm{ST}$ & $\mathrm{CT}$ & $\mathrm{ST}$ & $\mathrm{CT}$ \\
\hline 1 & $\begin{array}{l}\text { Neochlorogenic } \\
\text { acid }\end{array}$ & 0.68 & 3.69 & 1.75 & 3.25 & 3.59 \\
\hline 2 & Chlorogenic acid & 2.00 & 3.73 & 1.61 & 3.87 & 4.31 \\
\hline 3 & Caffeic acid & 2.44 & 3.02 & 2.97 & 4.11 & 4.46 \\
\hline 4 & $\begin{array}{l}\text { Cryptochlorogenic } \\
\text { acid }\end{array}$ & 0.68 & 3.38 & 2.41 & 3.47 & 3.22 \\
\hline 5 & $1,3-$ & 3.99 & 1.58 & 2.95 & 1.91 & 3.44 \\
\hline & $\begin{array}{c}\text { Dicaffeoylquinic } \\
\text { acid }\end{array}$ & & & & & \\
\hline 6 & $\begin{array}{l}\text { Luteolin-7- } O-\beta-\mathrm{D}- \\
\text { glucoside }\end{array}$ & 2.12 & 1.89 & 0.64 & 3.31 & 1.30 \\
\hline 7 & $\begin{array}{c}\text { Isochlorogenic acid } \\
\text { B }\end{array}$ & 1.16 & 3.66 & 1.96 & 2.48 & 0.46 \\
\hline 8 & $\begin{array}{c}\text { Isochlorogenic acid } \\
\text { A }\end{array}$ & 3.53 & 2.93 & 2.12 & 3.83 & 2.67 \\
\hline 9 & $\begin{array}{c}\text { Apigenin-7- } O-\beta-\mathrm{D}- \\
\text { glucoside }\end{array}$ & 1.52 & 1.77 & 2.27 & 1.05 & 3.80 \\
\hline 10 & $\begin{array}{c}\text { Isochlorogenic acid } \\
\text { C }\end{array}$ & 3.79 & 4.75 & 3.46 & 4.14 & 4.95 \\
\hline 11 & $\begin{array}{l}\text { Diosmetin-7- } \\
\text { glucoside }\end{array}$ & 2.49 & 3.57 & 2.00 & 4.12 & 2.48 \\
\hline 12 & Eriodictyol & 1.27 & 3.91 & 1.40 & 1.42 & 1.78 \\
\hline 13 & Linarin & 1.46 & 0.46 & 1.10 & 4.50 & 3.58 \\
\hline 14 & Tilianin & 4.27 & 4.02 & 4.13 & 0.84 & 1.10 \\
\hline 15 & Apigenin & 2.15 & 3.08 & 2.18 & 4.41 & 4.14 \\
\hline 16 & Diosmetin & 1.04 & 3.56 & 3.29 & 3.81 & 3.37 \\
\hline 17 & Hesperetin & 1.15 & 1.40 & 1.05 & 1.72 & 2.11 \\
\hline 18 & Acacetin & 1.69 & 1.32 & 0.36 & 2.17 & 1.50 \\
\hline
\end{tabular}




\begin{tabular}{lllllll}
\hline 19 & Luteolin & 3.31 & 4.48 & 2.55 & 4.12 & 4.38 \\
\hline
\end{tabular}

Total flavonoids, total phenolic acid content

Chrysanthemum leaf water extract and alcohol extract of the measured including Neochlorogenic acid, Chlorogenic acid, Caffeic acid, Cryptochlorogenic acid, 1,3Dicaffeoylquinic acid, Isochlorogenic acid B, Isochlorogenic acid A, Isochlorogenic acid $\mathrm{C}$, eight kinds of phenolic acids and Luteolin-7- $O-\beta$-D-glucoside, Apigenin-7- $O$ $\beta$-D-glucoside, Diosmetin-7-glucoside, Eriodictyol, Linarin, Tilianin, Apigenin, Diosmetin, Hesperetin, Acacetin, Luteolin and 11 kinds of flavonoids, a total of 19 components were detected.

The results showed that both water and alcohol extracts of chrysanthemum stem and leaf were rich in flavonoids and phenolic acids. The 11 flavonoids in the alcohol extract of chrysanthemum stem and leaf were higher than those in the water extract of chrysanthemum stem and leaf, the content of isochlorogenic acid A, B and C was higher than that of water extract from chrysanthemum stem and leaf, but the content of total phenolic acid was higher. Table 8 shows the contents of components in ethanol extract (CT) and water extract (ST) of chrysanthemum stem and leaf.

The neutral polysaccharide content in chrysanthemum stem and leaf was determined by phenol-sulfuric acid method, and the acid polysaccharide content in chrysanthemum stem and leaf was determined by carbazol-sulfuric acid method. The content of neutral polysaccharide was $(74.36 \pm 4.51) \mathrm{mg} / \mathrm{g}$, acid polysaccharide was $(26.72 \pm 1.84) \mathrm{mg} / \mathrm{g}$, and total polysaccharide was $(101.08 \pm 5.86) \mathrm{mg} / \mathrm{g}$. The above results showed that neutral and acidic polysaccharides were important components of total polysaccharides in chrysanthemum stems and leaves, and the content of neutral polysaccharides was higher.

Table 8 Content determination of 19 phenolic acids and flavonoids $(\mu \mathrm{g} / \mathrm{g})$

\begin{tabular}{ccc}
\hline Analytes & ST & CT \\
\hline Neochlorogenic acid & $0.71 \pm 0.09^{a}$ & $0.38 \pm 0.07$ \\
Chlorogenic acid & $1.70 \pm 0.15$ & $1.49 \pm 0.12$ \\
Caffeic acid & $0.09 \pm 0.01$ & $0.09 \pm 0.01$ \\
\hline
\end{tabular}




\begin{tabular}{|c|c|c|}
\hline Cryptochlorogenic acid & $1.06 \pm 0.10$ & $0.75 \pm 0.13$ \\
\hline 1,3-Dicaffeoylquinic acid & $7.62 \pm 1.37$ & $7.16 \pm 0.57$ \\
\hline $\begin{array}{l}\text { Luteolin-7- } O-\beta-\mathrm{D}- \\
\text { glucoside }\end{array}$ & $0.19 \pm 0.03$ & $1.13 \pm 0.05$ \\
\hline Isochlorogenic acid B & $0.16 \pm 0.03$ & $0.54 \pm 0.09$ \\
\hline Isochlorogenic acid A & $4.56 \pm 0.52$ & $11.96 \pm 0.87$ \\
\hline $\begin{array}{l}\text { Apigenin-7- } O-\beta-\mathrm{D}- \\
\text { glucoside }\end{array}$ & $0.08 \pm 0.01$ & $0.49 \pm 0.03$ \\
\hline Isochlorogenic acid C & $23.28 \pm 1.99$ & $41.58 \pm 5.42$ \\
\hline Diosmetin-7-glucoside & $12.46 \pm 0.74$ & $34.98 \pm 1.62$ \\
\hline Eriodictyol & $0.05 \pm 0.01$ & $0.08 \pm 0.01$ \\
\hline Linarin & $15.29 \pm 0.76$ & $45.10 \pm 2.36$ \\
\hline Tilianin & $8.40 \pm 4.34$ & $31.90 \pm 8.53$ \\
\hline Apigenin & $0.05 \pm 0.04$ & $1.00 \pm 0.13$ \\
\hline Diosmetin & $0.24 \pm 0.08$ & $3.72 \pm 0.31$ \\
\hline Hesperetin & $0.06 \pm 0.01$ & $0.30 \pm 0.01$ \\
\hline Acacetin & $0.36 \pm 0.03$ & $20.17 \pm 0.41$ \\
\hline Luteolin & $0.03 \pm 0.01$ & $0.24 \pm 0.08$ \\
\hline $\mathrm{TP}^{b}$ & $5958.47 \pm 463.63$ & $8739.31 \pm 727.90$ \\
\hline $\mathrm{TF}$ & $5657.36 \pm 752.48$ & $19014.80 \pm 1399.61$ \\
\hline TPF & $11615.83 \pm 950.99$ & $27754.11 \pm 1548.51$ \\
\hline
\end{tabular}

${ }^{a}$ Mean $\pm \mathrm{SD}(\mathrm{n}=10)$.

${ }^{b} \mathrm{TP}$ is the total content of phenolic acids; TF is the total content of flavonoids; TPF is the total content of TP and TF.

\section{Discussion}

The pathogenesis of IBD is still unclear. At present, corticosteroids, aminosalicylic acid, sulfasalazine and immunosuppression ${ }^{33}$ are the main drugs and means for the treatment of IBD. These drugs can significantly reduce the inflammatory response and improve the clinical symptoms of patients, but long-term use still has some side effects, 
and the price of drugs is expensive, resulting in the quality of life and satisfaction of patients greatly reduced. In recent years, there have been some new preparations and therapies, such as biological preparations ${ }^{34}$ and fecal bacteria transplantation ${ }^{35}$, but no breakthrough has been made.

Previously, TAO et al. ${ }^{18,20}$ showed that chrysanthemum polysaccharide could improve ulcerative colitis by promoting the growth of beneficial intestinal flora, regulating intestinal microecological balance, and restoring the immune system, while significantly reducing the levels of inflammatory cytokines TNF- $\alpha$, IL-6, IFN- $\gamma$ and IL$1 \beta$. As a non-medicinal part of chrysanthemum, the yield of chrysanthemum stem and leaf was significantly higher than that of chrysanthemum, but it could not be effectively used, resulting in resource waste. In this study, we first evaluated the anti-inflammatory and antioxidant effects of water and alcohol extracts from chrysanthemum stem and leaf using the zebrafish IBD model. Zebrafish digestive systems develop rapidly, starting to take shape about 18 hours after fertilization. Two to three days after fertilization, the young fish hatch and form a continuous intestine. By $5 \mathrm{dpf}$, the digestive tract supports feeding and digestion, and many digestive organs, such as the intestine, liver, gall bladder, and pancreas, are functionally similar to their mammalian counterparts $^{36}$. According to the results, the IBD model of zebrafish induced by 0.5\%DSS strongly accumulated intestinal acidic mucin, enlarged intestinal lumen, and damaged intestinal mucosa, while chrysanthemum stem and leaf extract inhibited the up-regulation of various inflammatory factors (IL-1 $\beta$, IL-8, MMP9) to varying degrees, showing its effective role in inflammatory regulation. In addition, the antioxidant function can be achieved by up-regulating superoxide dismutase activity. The antiinflammatory effect of water extract of chrysanthemum stem and leaf group increased with the decrease of concentration, while the anti-inflammatory effect of alcohol extract group was the best at medium concentration, and the antioxidant effect of both extracts was good at high concentration.

Simultaneously, a UPLC-TQ/MS method for the determination of water extract and alcohol extract from chrysanthemum stem and leaf was established and verified. The results show that the method is accurate and reliable. Suitable for the chrysanthemum 
leaf of Neochlorogenic acid, Chlorogenic acid, Caffeic acid, Cryptochlorogenic acid, 1,3-Dicaffeoylquinic acid, Isochlorogenic acid B, Isochlorogenic acid A, Isochlorogenic acid $\mathrm{C}$, eight kinds of phenolic acids and Luteolin-7- $O-\beta$-D-glucoside, Apigenin-7-O- $\beta$-D-glucoside, Diosmetin-7-glucoside, Eriodictyol, Linarin, Tilianin, Apigenin, Diosmetin, Hesperetin, Acacetin, Luteolin and 11 kinds of flavonoids determination. In addition, neutral and acidic polysaccharides were determined by phenol-sulfuric acid method and carbazol-sulfuric acid method, and the total polysaccharide content in chrysanthemum stems and leaves was obtained. The contents of total flavonoids and phenolic acids in the alcohol extract of chrysanthemum stem and leaf were significantly higher than those in the water extract of chrysanthemum stem and leaf, but the water-soluble polysaccharides were significantly more in the water extract of chrysanthemum stem and leaf.

In this present experimental results showed that different extracts from chrysanthemum stem and leaf have certain anti-inflammatory and antioxidant effects, and the main bioactive components were analyzed including flavonoids, phenolic acid and polysaccharides. However, the anti-inflammatory and anti-oxidation mechanisms and targets of these bioactive components need to be further studied and explored.

\section{Conclusion}

In summary, in response to the need for the development of therapeutic agents for IBD, our study confirmed the anti-inflammatory and antioxidant effects of Chrysanthemum morifolium leaf extract in a zebrafish inflammatory bowel disease model, and determined its flavonoid, phenolic acid and polysaccharide components. This study may also be helpful to improve the utilization of chrysanthemum stem and leaf resources.

\section{Abbreviations}

DSS: sodium glucan sulfate; IBD: Inflammatory bowel disease; TCM: Traditional Chinese medicine; ST: Water extract from stem and leaf of chrysanthemum; CT: Alcohol extract from stem and leaf of chrysanthemum; dpf: days postfertilization; SOD: Superoxide dismutase; IL: Interleukin; mmp9: Matrix metallopeptidase 9. 


\section{Declarations}

\section{Acknowledgements}

The authors are grateful for the support from Jiangsu Collaborative Innovation Center of Chinese Medicinal Resources Industrialization, National and Local Collaborative Engineering Center of Chinese Medicinal Resources Industrialization and Formulae Innovative Medicine, and Jiangsu Key Laboratory for High Technology Research of TCM Formulae.

\section{Ethics approval and consent to participate}

All zebrafish experiments were conducted according to the guidelines of Animal Ethics Committee of the Laboratory Animal Center, Nanjing University of Traditional Chinese Medicine.

\section{Consent for publication}

Not applicable.

\section{Availability of data and materials}

The datasets used during the current study are available from the corresponding author on reasonable request.

\section{Competing interests}

We wish to confirm that there are no known conflicts of interest associated with this publication and there has been no significant financial support for this work that could have influenced its outcome.

\section{Funding}

This work was supported by the National Natural Science Foundation of China (No.81603273), and the Science and Technology Program of Jiangsu Province, China (Social Development) (BE2019722).

\section{Authors' contributions}

Conception and design: LY and SSL. Acquisition of data: LY, LXJ, YH,and QDW. 
Analysis and interpretation of data: LY, GS. Writing of the manuscript: LY, SSL, and DJA. All authors read and approved the final manuscript. 


\section{References}

1. Podolsky D. Inflammatory bowel disease. The New England journal of medicine. 2002;347:417-29.

2. Rubin D, Ananthakrishnan A, Siegel C, Sauer B, Long M. ACG Clinical Guideline: Ulcerative Colitis in Adults. The American journal of gastroenterology. 2019;114:384-413.

3. Rubin D, Sninsky C, Siegmund B, Sans M, Hart A, Bressler B, et al. International Perspectives on Management of Inflammatory Bowel Disease: Opinion Differences and Similarities Between Patients and Physicians From the IBD GAPPS Survey. Inflammatory bowel diseases. 2021.

4. Molodecky N, Soon I, Rabi D, Ghali W, Ferris M, Chernoff G, et al. Increasing incidence and prevalence of the inflammatory bowel diseases with time, based on systematic review. Gastroenterology. 2012;142:46-54.e42; quiz e30.

5. Stulman M, Asayag N, Focht G, Brufman I, Cahan A, Ledderman N, et al. Epidemiology of Inflammatory Bowel Diseases in Israel: A Nationwide Epi-Israeli IBD Research Nucleus Study. Inflammatory bowel diseases. 2021.

6. Kaplan G. The global burden of IBD: from 2015 to 2025. Nature reviews. Gastroenterology \& hepatology. 2015;12:720-7.

7. Kudelka M, Stowell S, Cummings R, Neish A. Intestinal epithelial glycosylation in homeostasis and gut microbiota interactions in IBD. Nature reviews. Gastroenterology \& hepatology. 2020;17:597-617.

8. Jergens A, Parvinroo S, Kopper J, Wannemuehler M. Rules of Engagement: Epithelial-Microbe Interactions and Inflammatory Bowel Disease. Frontiers in medicine. 2021;8:669913.

9. Somani S, Modi K, Majumdar A, Sadarani B. Phytochemicals and their potential usefulness in inflammatory bowel disease. Phytotherapy research : PTR. 2015;29:339-50.

10. Pagnini C, Cominelli F. Tumor Necrosis Factor's Pathway in Crohn's Disease: Potential for Intervention. International journal of molecular sciences. 2021;22.

11. Burisch J, Bergemalm D, Halfvarson J, Domislovic V, Krznaric Z, Goldis A, et al. The use of 5 aminosalicylate for patients with Crohn's disease in a prospective European inception cohort with 5 years follow-up - an Epi-IBD study. United European gastroenterology journal. 2020;8:949-60. 12. López-Sanromán A. Steroids and Postoperative Complications in IBD. Current drug targets. 2019;20:1323-6.

13. Bernstein C. Past Time for Doctors to Lessen their Dependence on Corticosteroids in the Treatment of IBD. The American journal of gastroenterology. 2018;113:418-20.

14. Wan $\mathrm{P}$, Chen $\mathrm{H}$, Guo $\mathrm{Y}$, Bai A. Advances in treatment of ulcerative colitis with herbs: from bench to bedside. World journal of gastroenterology. 2014;20:14099-104.

15. Yang L, Luo H, Tan D, Zhang S, Zhong Z, Wang S, et al. A recent update on the use of Chinese medicine in the treatment of inflammatory bowel disease. Phytomedicine : international journal of phytotherapy and phytopharmacology. 2021;92:153709.

16. Luyen B, Tai B, Thao N, Cha J, Lee $\mathrm{H}$, Lee $\mathrm{Y}$, et al. Anti-inflammatory components of Chrysanthemum indicum flowers. Bioorganic \& medicinal chemistry letters. 2015;25:266-9.

17. Wang J, Liang Q, Zhao Q, Tang Q, Ahmed A, Zhang Y, et al. The effect of microbial composition and proteomic on improvement of functional constipation by Chrysanthemum morifolium polysaccharide. Food and chemical toxicology : an international journal published for the British Industrial Biological Research Association. 2021;153:112305.

18. Tao J, Duan J, Jiang S, Feng N, Qiu W, Ling Y. Chrysanthemum morifoliumPolysaccharides 
from Ramat ameliorate colitis rats by modulating the intestinal microbiota community. Oncotarget. 2017;8:80790-803.

19. Tao J, Duan J, Jiang S, Guo J, Qian Y, Qian D. Simultaneous determination of six short-chain fatty acids in colonic contents of colitis mice after oral administration of polysaccharides from Chrysanthemum morifolium Ramat by gas chromatography with flame ionization detector. Journal of chromatography. B, Analytical technologies in the biomedical and life sciences. 2016:88-94.

20. Tao J, Duan J, Zhang W, Jiang S, Guo J, Wei D. Chrysanthemum morifoliumPolysaccharides From Ramat Ameliorate Colitis Rats via Regulation of the Metabolic Profiling and NF-K B/TLR4 and IL-6/JAK2/STAT3 Signaling Pathways. Frontiers in pharmacology. 2018;9:746.

21. Chen S, Liu J, Dong G, Zhang X, Liu Y, Sun W, et al. Flavonoids and caffeoylquinic acids in Chrysanthemum morifolium Ramat flowers: A potentially rich source of bioactive compounds. Food chemistry. 2021;344:128733.

22. Liang F, Hu C, He Z, Pan Y. An arabinogalactan from flowers of Chrysanthemum morifolium: structural and bioactivity studies. Carbohydrate research. 2014;387:37-41.

23. Kuang C, LV D, Shen G, Li S, Luo Q, Zhang Z. Chrysanthemum morifoliumChemical composition and antimicrobial activities of volatile oil extracted from Ramat. Journal of food science and technology. 2018;55:2786-94.

24. Sassi A, Skhiri F, Chraief I, Bourgougnon N, Hammami M, Aouni M. Essential oils and crude extracts from Chrysanthemum trifurcatum leaves, stems and roots: chemical composition and antibacterial activity. Journal of oleo science. 2014;63:607-17.

25. Chiang $Y, W u Y, L i n L, T s a i T$. Comparative biotransformation of luteolin and apigenin from the flower extract and the stem-and-leaf extract of Dendranthema morifolium Ramat. Tzvel. in rats. Journal of the science of food and agriculture. 2021.

26. G S, C W, N D, D K, F S. Production of clones of homozygous diploid zebra fish (Brachydanio rerio). Nature. 1981;291:293-6.

27. Howe K, Clark M, Torroja C, Torrance J, Berthelot C, Muffato M, et al. The zebrafish reference genome sequence and its relationship to the human genome. Nature. 2013;496:498-503.

28. Dubois M, Gilles KA, Hamilton JK, Rebers PA, Smith F. Colorimetric Method for Determination of Sugars and Related Substances. Anal Chem. 1956;28:350-6.

29. Li J, Kisara K, Danielsson S, Lindström M, Gellerstedt G. An improved methodology for the quantification of uronic acid units in xylans and other polysaccharides. Carbohydrate research. 2007;342:1442-9.

30. Yang L, Zhou X, Huang W, Fang Q, Hu J, Yu L, et al. Protective Effect of Phillyrin on Lethal LPS-Induced Neutrophil Inflammation in Zebrafish. Cellular physiology and biochemistry : international journal of experimental cellular physiology, biochemistry, and pharmacology. 2017;43:2074-87.

31. Westerfield M. The Zebrafish Book. A Guide for The Laboratory Use of Zebrafish (Danio rerio). zebrafish book a guide for the laboratory use of zebrafish. 2000.

32. Oehlers S, Flores M, Hall C, Crosier K, Crosier P. Retinoic acid suppresses intestinal mucus production and exacerbates experimental enterocolitis. Disease models \& mechanisms. 2012;5:457-67.

33. Bots S, Gecse K, Barclay M, D'haens G. Combination Immunosuppression in IBD. Inflammatory bowel diseases. 2018;24:539-45.

34. Danese S, Vuitton L, Peyrin-Biroulet L. Biologic agents for IBD: practical insights. Nature 
reviews. Gastroenterology \& hepatology. 2015;12:537-45.

35. Paramsothy S, Nielsen S, Kamm M, Deshpande N, Faith J, Clemente J, et al. Specific Bacteria and Metabolites Associated With Response to Fecal Microbiota Transplantation in Patients With Ulcerative Colitis. Gastroenterology. 2019;156:1440-54.e2.

36. Yang $\mathrm{Y}$, Tomkovich $\mathrm{S}$, Jobin $\mathrm{C}$. Could a swimming creature inform us on intestinal diseases? Lessons from zebrafish. Inflammatory bowel diseases. 2014;20:956-66. 